\title{
A LASER STRAIN GAUGE FOR ACCELERATOR TARGETS
}

\author{
A. Hassanein, J. Norem, ANL, Argonne, IL 60439
}

\begin{abstract}
Multi megawatt accelerators can deliver sufficient power to a target to destroy it in a few pulses. In order to look at the response of solid and liquid targets under these high power pulses, we are developing optical methods of measuring surface deformations with time resolutions limited by laser pulse lengths. These methods can be used to examine the surfaces of solids and liquids during elastic deformation and unstable hydrodynamic flows. We present preliminary results of a system designed for target tests using the Brookhaven AGS and the Argonne CHM linac.
\end{abstract}

\section{INTRODUCTION}

The next generation of particle accelerators planned for neutrino experiments[1], muon colliders or neutrino sources assume that beam powers can be raised to a few megawatts and this power can be deposited into a target with a cross section of a $\mathrm{cm}$ or two. Unfortunately this means that the power deposited will be on the order of the binding energy per atom, and these power levels can be highly destructive. An example of this is a calculation done for the muon based neutrino source which shows the target response to a $2 \mathrm{~ns}$ bunch hitting a carbon rod target $1.5 \mathrm{~cm}$ in diameter, Figure 1.

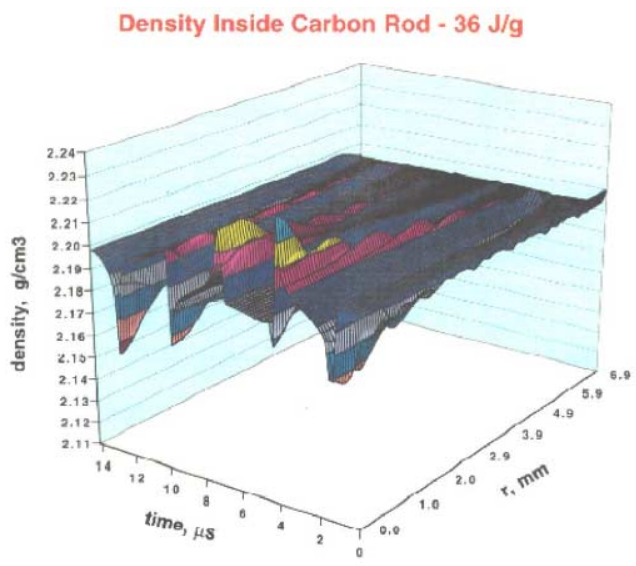

Figure 1, Response of a carbon target to a proton beam.

In Figure 1, the density, plotted vertically, is a function of the time, to the left, and radius, to the right. The rapid motion of shock waves reflecting from the center to the outside, with a period of about $0.25 \mathrm{MHz}$, is seen. This motion, while characteristic of all target behavior at this power level has not been experimentally verified, and there has, in fact, been little experimental study of this phenomenon.
While the physics of beam power deposition in solids is somewhat straightforward, many studies assume the target will be a liquid metal, perhaps a liquid jet, which would absorb the beam power while mechanically unconfined. The physics of beams hitting liquids is more complex than the case of solids. A variety of effects such as cavitation, deformation, and Raleigh-Taylor instabilities will occur, complicating the final state. These effects are being studied and simulated.

In order to compare these simulations with data, an experiment is being done at the Brookhaven AGS, with beams of $10^{12}-10^{13}$ protons in a $\sigma=\sim 10 \mathrm{~ns}$ pulse, as part of E-951[2]. Data on beam interactions with carbon rods are being taken with fast strain gauges mounted on the outside. Data with a mercury jet is taken with a fast camera and pulsed light source. In addition, studies of the response of mercury in a pool have been done, also with a camera.

Since the lifetime of solid targets may be determined in part by fatigue, and the dynamics of the liquid targets are determined by the structure of the deformation, we are developing optical diagnostics based on engineering methods used for optical testing.

\section{SCHLIEREN AND RONCHI OPTICS}

The basic optical system used in a Schlieren or Ronchi optical system is shown in Figure 2.

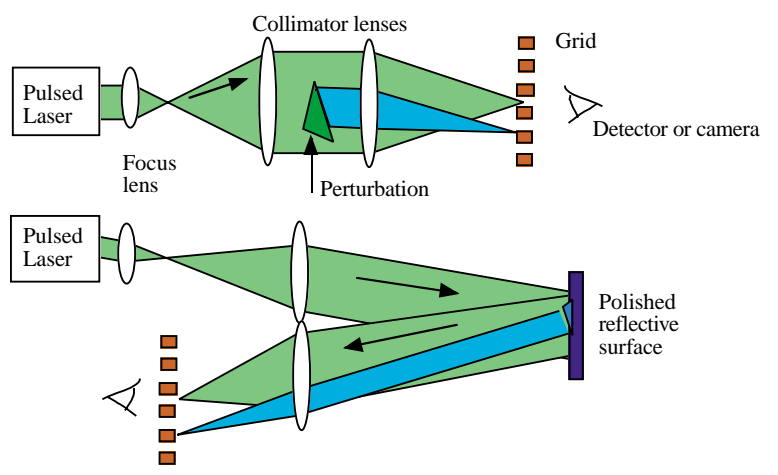

Figure 2, The Schlieren Ronchi optical systems.

The Schlieren optical system is a method of producing dark field illumination of a transparent sample, which will permit refractive (or reflective) perturbations to be preferentially illuminated. Using a modification using grids, proposed by Ronchi[3], it is possible to make quantitative measurements, since the transmitted light will be modulated by the magnitude of the refractive or reflective perturbation. This makes it possible to make quantitative measurements. 
The sensitivity of this system is limited by diffraction. The magnitude of the beam induced effects can be evaluated by looking at simulations like those in Figure 1, which might set the minimum measurable strain of a few microns, with a maximum given by one pulse demolition of the target. Since the velocities of sound in condensed matter is $1000-5000 \mathrm{~m} / \mathrm{s}$ and the dimensions of the anticipated beams is $1-2 \mathrm{~mm}$, the time scale for the stresses and strains is on the order of $1 \mu \mathrm{s}$.

\section{EXPERIMENTAL DEMONSTRATION}

We have constructed a prototype Schlieren / Ronchi setup and are in the process of testing it with proton and electron beams. A preliminary test of the system using the BNL AGS external proton beam parasitic to the E-951 experimental apparatus was unsuccessful. The proton beam interacted with the CCTV camera immediately following every proton pulse for a time long enough for the thermal effects to decay and no deflections were seen.

Initial tests using the $20 \mathrm{MeV}$ electron beam from the ANL-CHM linac have shown large deformations of a metal target. These tests were made with a $2 \mathrm{~mW} 650 \mathrm{~nm}$ diode laser. The metal mirrors were aligned at an angle to the beam, and the knife edge was oriented horizontally, rather than vertically as sketched in Figure 3. We have looked at transparent glass targets, but these began to brown before showing significant deformations. (Quartz, while not browning, would show a much smaller thermal expansion.)
The initial images, seen in Figure 4, show 5 pulses of $10^{12}$ electrons hitting the mirror and deflecting it. The deflection lasts for a time consistent with the thermal constant of the material and is asymmetrically distributed around the beam center. Immediate plans include improved optics, a better camera and image processing software. The use of a YAG laser instead of a cw diode laser will permit timing to be done at the level of a few ns.

\section{SUMMARY}

The next generation of accelerators will be able to produce more power than existing target technology can survive. We are developing sensitive instrumentation which can look at the response of solid and liquid targets to high power beam pulses with good spatial and time resolution. Initial results are encouraging.

\section{ACKNOWLEDGEMENTS}

This work was supported in part by the US Dept. of Energy and Illinois Consortium for Accelerator Research. P. Thieberger N. Simos helped at BNL, and C. Jonah and Lauren Ducas helped with the Argonne test.

\section{REFERENCES}

[1] http://www.cap.bnl.gov/mumu/studyii/FS2-report.html

[2] http://www.hep.princeton.edu/ mcdonald/mumu /target/targettrans29a.ps

[3] V. Ronchi, La Prova dei Sistema Ottica, Bologna, 1925

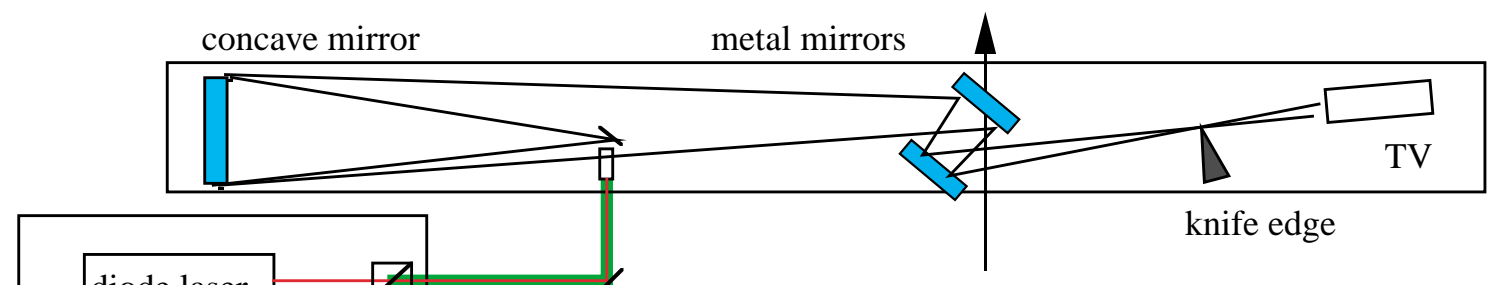

electron beam

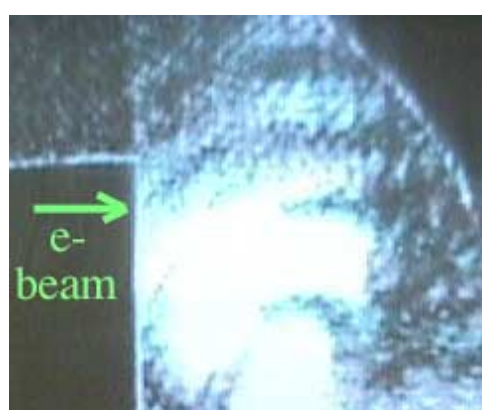

Figure 3, The experimental setup at the ANL/CHM linac.

Figure 4, Very preliminary data showing deformations from an electron beam. 